\title{
Conjugate Addition of Mixed Organozinc Compounds and Functionalized Organozinc Cuprates to Nitroolefins
}

\author{
Audrius Rimkus and Norbert Sewald* \\ University of Bielefeld, Faculty of Chemistry, PO Box 1001 31, D-33501 Bielefeld, Germany \\ norbert.sewald@uni-bielefeld.de
}

Supporting Information

\section{General remarks:}

${ }^{1} \mathrm{H}$ NMR and ${ }^{13} \mathrm{C}$ NMR spectra were recorded on a Gemini 200 (Varian) spectrometer $\left({ }^{1} \mathrm{H}: 200\right.$ $\mathrm{MHz},{ }^{13} \mathrm{C}: 50 \mathrm{MHz}$ ), Gemini 2000 (Varian) spectrometer $\left({ }^{1} \mathrm{H}: 200 \mathrm{MHz},{ }^{13} \mathrm{C}: 50 \mathrm{MHz},{ }^{31} \mathrm{P}: 81\right.$ $\mathrm{MHz})$ or on an AC 250-P (Bruker) spectrometer $\left({ }^{1} \mathrm{H}: 250 \mathrm{MHz},{ }^{13} \mathrm{C}\right.$ : $\left.63 \mathrm{MHz}\right)$. NMR spectra were recorded using solutions in deuterated chloroform $\left(\mathrm{CDCl}_{3}\right)$, with TMS as the internal standard, and shift values are reported in parts per million (ppm). Abbreviations for signal coupling are as follows: s, singlet; $d$, doublet; $t$, triplet; q, quartet; m, multiplet. IR spectra were collected on a FT/IR-410 (Jasco) spectrometer from the neat oil film (sodium chloride plates) or from a solid in potassium bromide pellet. Peaks are reported in $\mathrm{cm}^{-1}$ with following abbreviations for signal intensity: s, strong; m, medium; w, weak; br., broad. Mass spectra were recorded on GCMS-QP5050A (Shimadzu) gas chromatograph/mass spectrometer using a HP-5MS (25 m, $0.25 \mathrm{~mm}$ ID, $0.33 \mu \mathrm{m}$, Hewlett Packard) column. Isobutane was used as reagent gas for CI measurements. Elemental analyses were performed on CHNS-932 (Leco) apparatus. Capillary gas chromatography was performed on a GC-17A (Shimadzu) gas chromatograph using a HP5MS (25 m, $0.25 \mathrm{~mm} \mathrm{ID,} 0.33 \mu \mathrm{m}$, Hewlett Packard) column. Analytical TLC was performed on silica gel plates $60 \mathrm{~F}_{254}$ (Merck); compounds were visualized by illumination with UV light, staining with iodine or dipping the plates in a cerium sulfate-ammonium molybdate solution, followed by heating. Flash column chromatography was carried out with silica gel $60(40-63 \mu \mathrm{m})$ from Merck. Melting points were measured on Melting Point B-540 (Büchi) apparatus and are uncorrected.

All reactions were performed under an inert atmosphere (argon or nitrogen). Tetrahydrofuran (THF) and diethyl ether $\left(\mathrm{Et}_{2} \mathrm{O}\right)$ were distilled from sodium benzophenone ketyl immediately prior to use. Petrol ether ( $\mathrm{PE}, 30-60{ }^{\circ} \mathrm{C}$ boiling point fraction) and ethyl acetate (EtOAc) were used for flash chromatography and were distilled over $\mathrm{CaCl}_{2}$. The following compounds were commercially available and used without further purification: diethylzinc (Fluka), zinc powder (-100 mesh, $99.998 \%$ purity, Aldrich); copper(II) triflate (Aldrich), trimethylsilylmethyllithium solution (1 M in pentane; Fluka), trimethylsilyl bromide (Acros). Anhydrous LiCl (Aldrich) was dried with a heatgun at 0.01 mbar before use.

Trimethylsilyl chloride, dibromoethane and 1-methyl-2-pyrrolidone (NMP) were distilled prior to use. 


\section{General experimental procedure for conjugate addition of alkyl(trimethylsilylmethyl)zinc compounds to nitroolefins:}

1,2-Dibromoethane $(0.07 \mathrm{~mL}, 0.8 \mathrm{mmol})$ was added dropwise to a stirred mixture of zinc dust $(0.52 \mathrm{~g}, 8.0 \mathrm{mmol})$ in THF $(2.5 \mathrm{~mL})$ at RT under argon, whilst heating with a heat gun to boil the solvent gently. After ca. $1 \mathrm{~min}$ the reaction mixture foams and the heating is interrupted. After 1-3 min, this heating-cooling procedure is repeated two more times. Upon complete addition the mixture was cooled to RT and then trimethylsilyl chloride $(0.07 \mathrm{~mL}, 0.55 \mathrm{mmol})$ was added dropwise over $5 \mathrm{~min}$, again with gentle heating. After complete addition, the mixture was stirred at RT for further $5 \mathrm{~min}$. THF solutions $(1.5 \mathrm{~mL})$ of the appropriate iodo derivative $(2.0 \mathrm{mmol})$ and of dodecane (ca. $0.4 \mathrm{~mL}$ ) as internal standard for $\mathrm{GC}$ analysis were added dropwise over $5 \mathrm{~min}$, and then the reaction was stirred at $50{ }^{\circ} \mathrm{C}$ for $2-4 \mathrm{~h}$. The zinc insertion reaction was monitored by GC analysis. When the reaction was complete, the reaction mixture was cooled to RT and the excess zinc dust allowed to settle for $15 \mathrm{~min}$. The pale grey solution was transferred to a flame dried flask that contained a solution of copper salt $(0.05 \mathrm{mmol}, 5 \mathrm{~mol} \%$ referring to nitroolefin) in THF $(2.0 \mathrm{~mL})$ cooled to $-40^{\circ} \mathrm{C}$. A solution of trimethylsilylmethyllithium $(1 \mathrm{M}$ in pentane, $2.0 \mathrm{~mL}, 2.0 \mathrm{mmol}$,) was added dropwise to the zinc iodide solution. and then stirred at $-40{ }^{\circ} \mathrm{C}$ for $1 \mathrm{~h}$. NMP $(0.33 \mathrm{~mL}, 3.4 \mathrm{mmol})$, trimethylsilyl bromide $(0.35 \mathrm{~mL}, 2.7 \mathrm{mmol})$ and then nitroolefin $(1.1 \mathrm{mmol})$ in THF $(1.5 \mathrm{~mL})$ were subsequently added dropwise at $-40{ }^{\circ} \mathrm{C}$. The reaction was allowed to warm up to $-30^{\circ} \mathrm{C}$, stirred at this temperature for $3 \mathrm{~h}$ and finally allowed to warm up slowly to RT overnight. The reaction mixture was poured into saturated ammonium chloride solution $(10 \mathrm{~mL})$ and stirred at $\mathrm{RT}$ for $10 \mathrm{~min}$. The resulting solution was extracted with diethyl ether $(3 \times 30 \mathrm{~mL})$. The combined extracts were washed with brine $(30 \mathrm{~mL})$, dried $\left(\mathrm{MgSO}_{4}\right)$ and concentrated in vacuo. The crude residue was purified by column chromatography.

\section{(1-Cyclohexyl-2-nitroethyl)-benzene (4a)}

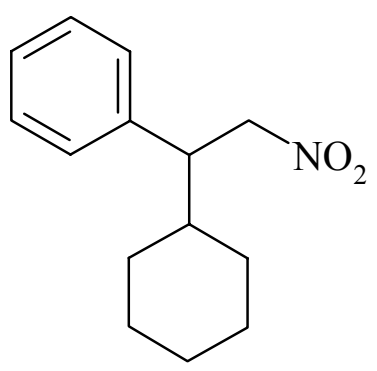

White crystals

Melting point:

$64-65{ }^{\circ} \mathrm{C}$

Molecular weight:

$233.31\left(\mathrm{C}_{14} \mathrm{H}_{19} \mathrm{NO}_{2}\right)$

Yield:

$174 \mathrm{mg}(70 \%)$

${ }^{1} \mathrm{H}-\mathrm{NMR}\left(200 \mathrm{MHz}, \mathrm{CDCl}_{3}\right): \quad \delta=0.77-1.88(11 \mathrm{H}, \mathrm{m}) ; 3.27(1 \mathrm{H}, \mathrm{ddd}, J=9.9 \mathrm{~Hz}, 8.0 \mathrm{~Hz}$, $5.9 \mathrm{~Hz}) ; 4.62(1 \mathrm{H}, \mathrm{dd}, J=12.3 \mathrm{~Hz}, 9.9 \mathrm{~Hz}) ; 4.80(1 \mathrm{H}, \mathrm{dd}$, $J=12.3 \mathrm{~Hz}, 5.9 \mathrm{~Hz}) ; 7.06-7.43(5 \mathrm{H}, \mathrm{m})$

${ }^{13} \mathrm{C}-\mathrm{NMR}\left(50 \mathrm{MHz}, \mathrm{CDCl}_{3}\right): \quad \delta=26.5,31.0,31.4,41.3,50.7,79.3,127.9,128.7,129.1$, 139.3 
MS:

IR ( $\mathrm{KBr})$ :

$\mathrm{R}_{\mathrm{f}}$ :

Elemental analysis (\%):
EI: $\mathrm{m} / \mathrm{z}=186$ (24\%); 105 (17\%); 104 (100\%); 91 (23\%); 83 (20\%); 55 (50\%); 41 (24\%) $\widetilde{v}=2915$ (s), 2852 (s), 1548 (s), 1442 (s), 1380 (s), 755 (s), $700(\mathrm{~s})$

$0.37\left(\mathrm{PE}+5 \% \mathrm{Et}_{2} \mathrm{O}\right)$

Calcd.: C 72.07; H 8.21; N 6.00

Found: C 72.32; H 7.91; N 5.98

\section{(E)-(2-Cyclohexylethenyl)-benzene (5a)}<smiles>C(=C/C1CCCCC1)\c1ccccc1</smiles>

Colourless oil

Molecular weight:

Yield:

${ }^{1} \mathrm{H}-\mathrm{NMR}\left(200 \mathrm{MHz}, \mathrm{CDCl}_{3}\right)$ :

${ }^{13} \mathrm{C}-\mathrm{NMR}\left(50 \mathrm{MHz}, \mathrm{CDCl}_{3}\right)$ :

MS:

IR (neat):

$\mathrm{R}_{\mathrm{f}}$ :

\section{1-Nitro-2-phenylnonane (4b)}

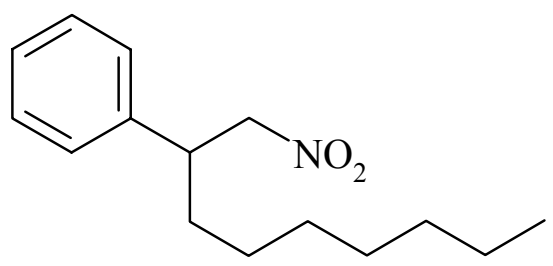

Light yellow oil

Molecular weight:

Yield:

${ }^{1} \mathrm{H}-\mathrm{NMR}\left(250 \mathrm{MHz}, \mathrm{CDCl}_{3}\right)$ :
$186.30\left(\mathrm{C}_{14} \mathrm{H}_{18}\right)$

$50 \mathrm{mg}(28 \%)$

$\delta=1.08-1.95 \quad(10 \mathrm{H}, \mathrm{m}) ; 2.18 \quad(1 \mathrm{H}, \mathrm{m}) ; 6.22(1 \mathrm{H}, \mathrm{dd}$, $J=6.6 \mathrm{~Hz}, 16.1 \mathrm{~Hz}) ; 6.40(1 \mathrm{H}, \mathrm{d}, J=16.1 \mathrm{~Hz}) ; 7.14-7.47(5 \mathrm{H}$, $\mathrm{m})$

$\delta=26.6,26.7,33.5,41.7,126.5,127.2,127.8,129.0,137.3$, 138.6

EI: $\mathrm{m} / \mathrm{z}=186(24 \%) ; 129$ (27\%); $128(23 \%) ; 117(10 \%)$; 115 (23\%); 105 (11\%); 104 (100\%); 91 (20\%)

$\widetilde{v}=3025$ (s), $2924(\mathrm{~s}), 2850(\mathrm{~s}), 1492(\mathrm{~m}), 1446$ (s), $963(\mathrm{~s})$, $742(\mathrm{~s}), 692(\mathrm{~s})$

$0.60(\mathrm{PE})$
$249.36\left(\mathrm{C}_{15} \mathrm{H}_{23} \mathrm{NO}_{2}\right)$

$64 \%(106 \mathrm{mg})$

$\delta=0.86(3 \mathrm{H}, \mathrm{m}) ; 1.11-1.35(10 \mathrm{H}, \mathrm{m}) ; 1.60-1.73(2 \mathrm{H}, \mathrm{m})$; $3.44(1 \mathrm{H}, \mathrm{m}) ; 4.47-4.61(2 \mathrm{H}, \mathrm{m}) ; 7.13-7.41(5 \mathrm{H}, \mathrm{m})$ 

${ }^{13} \mathrm{C}-\mathrm{NMR}\left(63 \mathrm{MHz}, \mathrm{CDCl}_{3}\right): \quad \delta=14.0,22.6,26.9,29.0,29.3,31.7,33.1,44.4,81.1,127.5$, $127.6,128.8,128.9,129.0,139.6$
MS:
CI: $\mathrm{m} / \mathrm{z}=273(13 \%) ; 272(69 \%) ; 219(11 \%) ; 218(16 \%)$; $217(21 \%) ; 216(100 \%) ; 214(35 \%) ; 203(16 \%) ; 189$ $(28 \%)$
IR (neat):
$\widetilde{v}=2927(\mathrm{~s}), 2856(\mathrm{~m}), 1554(\mathrm{~s}), 1454(\mathrm{w}), 1376(\mathrm{~m}), 1056$
$\mathrm{R}_{\mathrm{f}}$ : (w), $763(\mathrm{w}), 701(\mathrm{~m})$
$0.33\left(\mathrm{PE}+2 \% \mathrm{Et}_{2} \mathrm{O}\right)$

\section{(E)-1-Phenyl-1-nonene (5b)}

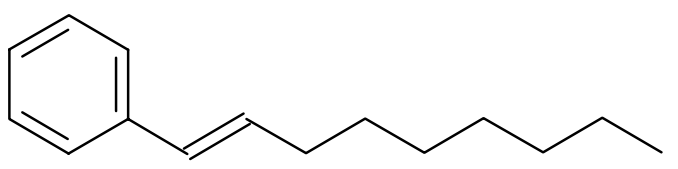

Colourless oil

Molecular weight:

Yield:

${ }^{1} \mathrm{H}-\mathrm{NMR}\left(250 \mathrm{MHz}, \mathrm{CDCl}_{3}\right)$ :

${ }^{13} \mathrm{C}-\mathrm{NMR}\left(63 \mathrm{MHz}, \mathrm{CDCl}_{3}\right)$ :

MS:

IR (neat):

$\mathrm{R}_{\mathrm{f}}$ :

Elemental analysis (\%):

\section{2-Phenyl-1-nitrohexane (4c)}<smiles>CCCCC(C[N+](=O)[O-])c1ccccc1</smiles>

Light yellow oil

Molecular weight:

Yield:

${ }^{1} \mathrm{H}-\mathrm{NMR}\left(200 \mathrm{MHz}, \mathrm{CDCl}_{3}\right.$ ):
$202.34\left(\mathrm{C}_{15} \mathrm{H}_{22}\right)$

$43 \%(176 \mathrm{mg})$

$\delta=0.89(3 \mathrm{H}, \mathrm{t}, J=7.0 \mathrm{~Hz}) ; 1.17-1.36(8 \mathrm{H}, \mathrm{m}) ; 1.32-1.57(2 \mathrm{H}$, $\mathrm{m})$; $2.21-2.27(2 \mathrm{H}, \mathrm{m}) ; 6.22(1 \mathrm{H}, \mathrm{dt}, J=15.8 \mathrm{~Hz}, 6.6 \mathrm{~Hz})$; $6.38(1 \mathrm{H}, \mathrm{d}, J=15.8 \mathrm{~Hz}) ; 7.12-7.40(5 \mathrm{H}, \mathrm{m})$ $\delta=14.1,22.7,29.2,29.4,29.7,31.9,33.1,125.9,126.7$, $128.5,129.7,131.3,138.0$

CI: $\mathrm{m} / \mathrm{z}=246(2 \%) ; 245$ (14\%); 204 (15\%); 203 (100\%); 202 (43\%); 201 (19\%); 190 (12\%); 189 (81\%); 175 (7\%); 141 (9\%); 133 (13\%); 119 (14\%); 117 (19\%); 105 (26\%) $\widetilde{v}=2954(\mathrm{~m}), 2923(\mathrm{~s}), 2854(\mathrm{~m}), 1461(\mathrm{w}), 964(\mathrm{w})$ 0.60 (PE)

Calcd.: C 89.04; H 10.96

Found: C 88.97; H 11.15 
${ }^{13} \mathrm{C}-\mathrm{NMR}\left(50 \mathrm{MHz}, \mathrm{CDCl}_{3}\right): \quad \delta=14.2,22.8,29.4,33.1,44.8,81.5,128.0,128.0,129.4$, 140.1

MS:

EI: $\mathrm{m} / \mathrm{z}=160(17 \%) ; 119(10 \%) ; 118(100 \%) ; 117(14 \%) ;$ 105 (11\%); 104 (23\%); $91(55 \%)$

IR (neat): $\quad \tilde{v}=2960(\mathrm{~m}), 2931(\mathrm{~m}), 2859(\mathrm{~m}), 1552(\mathrm{~s}), 1454(\mathrm{~m}), 1378$ (m), $763(\mathrm{~m}), 700(\mathrm{~s})$

Rf: $\quad 0.47$ (PE:EtOAc / 15:1)

Elemental analysis (\%): $\quad$ Calcd.: C $69.54 ; \mathrm{H} 8.27$; N 6.76

Found: C 69.59; H 8.17; N 6.79

\section{2-Cyclohexyl-1,1-dimethoxy-3-nitropropane (4d)}<smiles>COC(OC)C(C[N+](=O)[O-])C1CCCCC1</smiles>

Light yellow oil

Molecular weight:

Yield:

$231.29\left(\mathrm{C}_{11} \mathrm{H}_{21} \mathrm{NO}_{4}\right)$

${ }^{1} \mathrm{H}-\mathrm{NMR}\left(200 \mathrm{MHz}, \mathrm{CDCl}_{3}\right)$ :

$64 \mathrm{mg}(45 \%)$

$\delta=0.82-1.84(11 \mathrm{H}, \mathrm{m}) ; 2.53(1 \mathrm{H}, \mathrm{m}) ; 3.34(3 \mathrm{H}, \mathrm{s}) ; 3.36(3 \mathrm{H}$, s); $4.29(1 \mathrm{H}, \mathrm{dd}, J=13.5 \mathrm{~Hz}, 5.6 \mathrm{~Hz}) ; 4.35(1 \mathrm{H}, \mathrm{d}, J=4.8 \mathrm{~Hz})$; $4.51(1 \mathrm{H}, \mathrm{dd}, J=13.5 \mathrm{~Hz}, 6.4 \mathrm{~Hz})$

${ }^{13} \mathrm{C}-\mathrm{NMR}\left(50 \mathrm{MHz}, \mathrm{CDCl}_{3}\right): \quad \delta=26.7,26.8,26.9,30.0,31.1,37.6,45.8,54.8,56.0,74.3$, 105.4

MS:

EI: $\mathrm{m} / \mathrm{z}=153(5 \%) ; 75$ (100\%); 71 (10\%); 55 (9\%); 47

IR (neat): $(11 \%) ; 41(13 \%)$

$\widetilde{v}=2927(\mathrm{~s}), 2852(\mathrm{~s}), 1554(\mathrm{~s}), 1448(\mathrm{~m}), 1380(\mathrm{~m}), 1205$

$\mathrm{R}_{\mathrm{f}}$ : (m), $1130(\mathrm{~m}), 1068(\mathrm{~s}), 966(\mathrm{~m}), 732(\mathrm{~m})$

0.35 (PE:EtOAc / 15:1)

Elemental analysis (\%):

Calcd.: C 57.12; H 9.15; N 6.06

Found: C 57.18; H 9.04; N 6.03

\section{1,1-Dimethoxy-2-(nitromethyl)nonane (4e)}<smiles>CCCCCCCC(C[N+](=O)[O-])C(OC)OC</smiles> 
Colourless oil

Molecular weight:

Yield:

${ }^{1} \mathrm{H}-\mathrm{NMR}\left(250 \mathrm{MHz}, \mathrm{CDCl}_{3}\right)$ :

${ }^{13} \mathrm{C}-\mathrm{NMR}\left(63 \mathrm{MHz}, \mathrm{CDCl}_{3}\right)$ :

MS:

IR (neat):

Rf:

Elemental analysis (\%):
$247.34\left(\mathrm{C}_{12} \mathrm{H}_{25} \mathrm{NO}_{4}\right)$

$72 \%(120 \mathrm{mg})$

$\delta=0.88(3 \mathrm{H}, \mathrm{t}, J=6.6 \mathrm{~Hz}) ; 1.19-1.41(10 \mathrm{H}, \mathrm{m}) ; 1.57(2 \mathrm{H}, \mathrm{m})$; $2.53(1 \mathrm{H}, \mathrm{m}) ; 3.39(3 \mathrm{H}, \mathrm{s}) ; 3.40(3 \mathrm{H}, \mathrm{s}) ; 4.28(1 \mathrm{H}, \mathrm{d}$, $J=4.9 \mathrm{~Hz}) ; 4.29(1 \mathrm{H}, \mathrm{dd}, J=12.9 \mathrm{~Hz}, 6.6 \mathrm{~Hz}) ; 4.54(1 \mathrm{H}, \mathrm{dd}$, $J=12.8 \mathrm{~Hz}, 5.8 \mathrm{~Hz}$ )

$\delta=14.1,22.6,26.7,27.7,29.1,29.6,31.8,40.9,54.9,56.1$, $75.6,105.8$

CI: $\mathrm{m} / \mathrm{z}=216(32 \%) ; 214(54 \%) ; 182(26 \%) ; 170$ (11\%); $169(100 \%)$

$\widetilde{v}=2929(\mathrm{~s}) ; 2858(\mathrm{~s}) ; 1554(\mathrm{~s}) ; 1466(\mathrm{~m}) ; 1381(\mathrm{~s}) ; 1190$ (m); $1117(\mathrm{~s}) ; 1072(\mathrm{~s}) ; 970(\mathrm{w}) ; 723(\mathrm{w})$

$0.23\left(\mathrm{PE}+5 \% \mathrm{Et}_{2} \mathrm{O}\right)$

Calcd.: C 58.27; H 10.19; N 5.66

Found: C 58.54; H 10.28; N 5.61

\section{Methyl 2-(Nitromethyl)nonanoate (4f)}<smiles>CCCCCCCC(C[N+](=O)[O-])C(C)=O</smiles>

Colourless oil

Molecular weight:

Yield:

${ }^{1} \mathrm{H}-\mathrm{NMR}\left(250 \mathrm{MHz}, \mathrm{CDCl}_{3}\right)$ :

${ }^{13} \mathrm{C}-\mathrm{NMR}\left(63 \mathrm{MHz}, \mathrm{CDCl}_{3}\right)$ :

MS:

IR (neat):

$\mathrm{R}_{\mathrm{f}}$ :

Elemental analysis (\%):
$231.29\left(\mathrm{C}_{11} \mathrm{H}_{21} \mathrm{NO}_{4}\right)$

$46 \%(80 \mathrm{mg})$

$\delta=0.88(3 \mathrm{H}, \mathrm{t}, J=6.9 \mathrm{~Hz}) ; 1.14-1.48(9 \mathrm{H}, \mathrm{m}) ; 1.46-1.78(3 \mathrm{H}$, m); $3.20(1 \mathrm{H}, \mathrm{m}) ; 3.75(3 \mathrm{H}, \mathrm{s}) ; 4.41(1 \mathrm{H}, \mathrm{dd}, J=14.2,4.9)$; $4.73(1 \mathrm{H}, \mathrm{dd}, J=14.2,9.2)$

$\delta=14.0,22.6,26.6,28.9,29.2,29.3,31.7,43.0,52.3,75.2$, 172.8

CI: $\mathrm{m} / \mathrm{z}=255$ (5\%); 254 (34\%); 233 (4\%); $232(29 \%) ; 200$ (65\%); 198 (59\%); 196 (16\%); 186 (16\%); 185 (100\%); 173 (28\%); 142 (82\%); 141 (11\%); 140 (34\%) $\widetilde{v}=2959(\mathrm{w}), 2926(\mathrm{~m}), 2856(\mathrm{~m}), 1739(\mathrm{~s}), 1559(\mathrm{~s}), 1438$ (m), 1379 (s), $1251(\mathrm{w}), 1203(\mathrm{w}), 1174(\mathrm{w})$ 0.35 (PE:EtOAc / 10:1)

Calcd.: C 57.12; H 9.15; N 6.06

Found: C 57.82; H 9.09; N 5.72 


\section{Methyl 2-Cyclohexyl-3-nitropropanoate (4g)}<smiles>COC(=O)C(C[N+](=O)[O-])C1CCCCC1</smiles>

Colourless oil

Molecular weight:

$215.25\left(\mathrm{C}_{10} \mathrm{H}_{17} \mathrm{NO}_{4}\right)$

Yield:

$69 \%(453 \mathrm{mg})$

${ }^{1} \mathrm{H}-\mathrm{NMR}\left(250 \mathrm{MHz}, \mathrm{CDCl}_{3}\right): \quad \delta=0.97-1.36(5 \mathrm{H}, \mathrm{m}) ; 1.59-1.84(6 \mathrm{H}, \mathrm{m}) ; 3.11(1 \mathrm{H}, \mathrm{m}) ; 3.74$ $(3 \mathrm{H}, \mathrm{s}) ; 4.44(1 \mathrm{H}, \mathrm{dd}, J=14.6 \mathrm{~Hz}, 4.0 \mathrm{~Hz}) ; 4.79(1 \mathrm{H}, \mathrm{dd}$, $J=14.6 \mathrm{~Hz}, 10.5 \mathrm{~Hz})$

${ }^{13} \mathrm{C}-\mathrm{NMR}\left(63 \mathrm{MHz}, \mathrm{CDCl}_{3}\right): \quad \delta=25.9,26.1,26.2,30.4,30.5,38.5,48.7,52.2,73.9,172.4$

$\mathrm{MS}$ :

CI: $\mathrm{m} / \mathrm{z}=215(19 \%) ; 183(53 \%) ; 181(55 \%) ; 179(19 \%)$; $168(79 \%) ; 156(69 \%) ; 125(100 \%) ; 123(68 \%) ; 121$ $(39 \%) ; 111(17 \%) ; 109(17 \%)$

IR (neat): $\widetilde{v}=2931(\mathrm{~s}), 2854(\mathrm{~m}), 1735(\mathrm{~s}), 1558(\mathrm{~s}), 1438(\mathrm{~m}), 1376$ (m), $1253(\mathrm{w}), 1203(\mathrm{~m}), 1172(\mathrm{w}), 1041(\mathrm{w})$

$\mathrm{R}_{\mathrm{f}}$ : 0.45 (PE:EtOAc / 10:1)

Methyl 2-(Nitromethyl)octanoate (4h)

$\mathrm{MeOOC}$

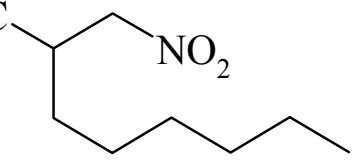

Colourless oil

Molecular weight:

$217.26\left(\mathrm{C}_{10} \mathrm{H}_{19} \mathrm{NO}_{4}\right)$

Yield:

$56 \%(131 \mathrm{mg})$

${ }^{1} \mathrm{H}-\mathrm{NMR}\left(250 \mathrm{MHz}, \mathrm{CDCl}_{3}\right)$ : $\delta=0.88(3 \mathrm{H}, \mathrm{t}, J=6.9 \mathrm{~Hz}) ; 1.17-1.42(8 \mathrm{H}, \mathrm{m}) ; 1.46-1.79(2 \mathrm{H}$, m); $3.19(1 \mathrm{H}, \mathrm{m}) ; 3.75(3 \mathrm{H}, \mathrm{s}) ; 4.42(1 \mathrm{H}, \mathrm{dd}, J=14.3 \mathrm{~Hz}$, $4.9 \mathrm{~Hz}) ; 4.74(1 \mathrm{H}, \mathrm{dd}, J=14.3 \mathrm{~Hz}, 9.5 \mathrm{~Hz})$

${ }^{13} \mathrm{C}-\mathrm{NMR}\left(50 \mathrm{MHz}, \mathrm{CDCl}_{3}\right): \quad \delta=14.0,22.5,26.6,28.9,29.3,31.5,42.9,52.4,75.2,172.8$ $\mathrm{MS}$ : CI: $\mathrm{m} / \mathrm{z}=241(1 \%) ; 240(13 \%) ; 219(13 \%) ; 218$ (100\%); $186(23 \%) ; 184(14 \%) ; 173(11 \%) ; 172$ (10\%); 171 $(36 \%) ; 139(7 \%) ; 128(5 \%)$

IR (neat): $\widetilde{v}=2954(\mathrm{~m}), 2931(\mathrm{~m}), 2858(\mathrm{~m}), 1739(\mathrm{~s}), 1558(\mathrm{~s}), 1438$

$\mathrm{R}_{\mathrm{f}}$ : (m), $1380(\mathrm{~m}), 1253$ (w), 1203 (m), 1176 (m), 1049 (w) 0.35 (PE:EtOAc / 10:1)

Elemental analysis (\%):

Calcd.: C 55.28; H 8.81; N 6.45

Found: C 55.07; H 8.95; N 6.76 


\section{General experimental procedure for conjugate addition of transmetallated functionalized diorganozinc compounds to nitroolefins:}

A mixture of an iodo derivative $(4.7 \mathrm{mmol})$ and neat diethylzinc $(2.95 \mathrm{~g}, 23.9 \mathrm{mmol})$ was stirred at $50{ }^{\circ} \mathrm{C}$ for $14 \mathrm{~h}$ (reaction monitoring by $\mathrm{GC}$ of hydrolyzed reaction aliquots). The ethyl iodide formed, and excess diethylzinc were removed in vacuo $\left(35^{\circ} \mathrm{C}, 1 \mathrm{~h}\right.$, ca. $\left.0.01 \mathrm{mbar}\right)$. The resulting oil was dissolved in dry THF $(4 \mathrm{~mL})$ and added to a THF solution $(5 \mathrm{~mL})$ of copper(I) cyanide $(211 \mathrm{mg}, 2.4 \mathrm{mmol})$ and lithium chloride $(199 \mathrm{mg}, 4.7 \mathrm{mmol})$ at $-20^{\circ} \mathrm{C}$. The resulting light-green solution was cooled to $-78^{\circ} \mathrm{C}$, and trimethylsilyl chloride $(598 \mathrm{mg}, 5.5 \mathrm{mmol})$ followed by a solution of nitroolefin $(2.3 \mathrm{mmol})$ in THF $(1.5 \mathrm{~mL})$ was added dropwise. The reaction mixture was slowly allowed to warm up to $-10^{\circ} \mathrm{C}$ overnight. Then ether was added $(20 \mathrm{~mL})$ and the mixture was quenched with saturated ammonium chloride solution $(10 \mathrm{~mL})$. The aqueous layer was extracted with ether $(2 \times 10 \mathrm{~mL})$. The combined organic layers were washed with brine $(10 \mathrm{~mL})$, dried $\left(\mathrm{MgSO}_{4}\right)$, and filtered. The solvent was evaporated, and the residual oil was purified by flash chromatography to afford the product.

\section{6-Nitro-5-phenylhexanenitrile (9a)}

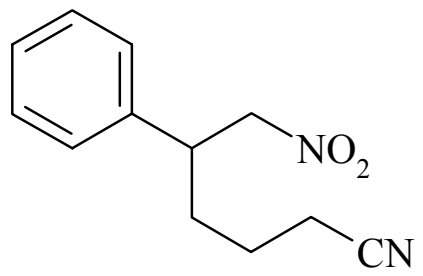

Colourless oil

Molecular weight:

Yield:

${ }^{1} \mathrm{H}-\mathrm{NMR}\left(250 \mathrm{MHz}, \mathrm{CDCl}_{3}\right)$

${ }^{13} \mathrm{C}-\mathrm{NMR}\left(63 \mathrm{MHz}, \mathrm{CDCl}_{3}\right)$ :

MS:

IR (neat):

$\mathrm{R}_{\mathrm{f}}$ :

Elemental analysis (\%):
$218.25\left(\mathrm{C}_{12} \mathrm{H}_{14} \mathrm{~N}_{2} \mathrm{O}_{2}\right)$

$67 \%(196 \mathrm{mg})$

$\delta=1.38-1.71 \quad(2 \mathrm{H}, \mathrm{m}) ; 1.72-1.98(2 \mathrm{H}, \mathrm{m}) ; 2.29(2 \mathrm{H}, \mathrm{t}$, $J=7.0 \mathrm{~Hz}) ; 3.48(1 \mathrm{H}, \mathrm{m}) ; 4.57(2 \mathrm{H}, \mathrm{d}, J=7.6 \mathrm{~Hz}) ; 7.07-7.52$ $(5 \mathrm{H}, \mathrm{m})$

$\delta=17.0,23.0,31.9,43.8,80.6,119.3,127.5,128.2,129.3$, 138.1

EI: $\mathrm{m} / \mathrm{z}=184(19 \%) ; 158(10 \%) ; 132(30 \%) ; 130(42 \%)$; 118 (44\%); 117 (54\%); 116 (91\%); 115 (24\%); 104 (31\%); 103 (36\%); 91 (100\%); 90 (21\%); 89 (49\%); 78 (28\%); 77 (39\%); 65 (24\%); 51 (24\%); 41 (22\%); 39 $(27 \%)$

$\widetilde{v}=2937(\mathrm{w}), 2246(\mathrm{w}), 1550(\mathrm{~s}), 1454(\mathrm{w}), 1430(\mathrm{w}), 1380$ (m), $1043(\mathrm{w}), 763(\mathrm{w}), 701(\mathrm{w})$

0.77 (PE:EtOAc / 3:1)

Calcd.: C 66.04; H 6.47; N 12.84

Found: C 66.12; H 6.23; N 12.79 


\section{Methyl 5-Cyano-2-(nitromethyl)pentanoate (9b)}<smiles>COC(=O)C(CCCC#N)C[N+](=O)[O-]</smiles>

Colourless oil

Molecular weight:

$200.19\left(\mathrm{C}_{8} \mathrm{H}_{12} \mathrm{~N}_{2} \mathrm{O}_{4}\right)$

Yield:

$66 \%(310 \mathrm{mg})$

${ }^{1} \mathrm{H}-\mathrm{NMR}\left(250 \mathrm{MHz}, \mathrm{CDCl}_{3}\right)$ :

$\delta=1.56-1.94(4 \mathrm{H}, \mathrm{m}) ; 2.41(2 \mathrm{H}, \mathrm{t}, J=6.6 \mathrm{~Hz}) ; 3.23(1 \mathrm{H}, \mathrm{m})$; $4.46(1 \mathrm{H}, \mathrm{dd}, J=14.3 \mathrm{~Hz}, 5.2 \mathrm{~Hz}) ; 4.76(1 \mathrm{H}, \mathrm{dd}, J=14.3 \mathrm{~Hz}$, $8.6 \mathrm{~Hz})$

${ }^{13} \mathrm{C}-\mathrm{NMR}\left(63 \mathrm{MHz}, \mathrm{CDCl}_{3}\right): \quad \delta=17.0,22.9,28.2,42.3,52.7,75.1,118.6,171.7$

MS:

CI: $\mathrm{m} / \mathrm{z}=202(10 \%) ; 201$ (100\%); 169 (41\%); 154 (11\%); $122(27 \%)$

IR (neat): $\quad \quad \widetilde{v}=2956(\mathrm{~s}), 2246(\mathrm{~m}), 1735(\mathrm{~s}), 1560(\mathrm{~s}), 1436(\mathrm{~s}), 1380(\mathrm{~s})$, $1207(\mathrm{~s}), 1058(\mathrm{~m}), 970(\mathrm{~m}), 736(\mathrm{w})$

$\mathrm{R}_{\mathrm{f}}$ : 0.48 (PE:EtOAc / 2:1)

Elemental analysis (\%):

Calcd.: C 48.00; H 6.04; N 14.00

Found: C 48.17; H 5.98; N 13.88

\section{1-Methyl 6-Ethyl 2-(Nitromethyl)hexanedioate (9c)}<smiles>CCOC(=O)CCCC(C[N+](=O)[O-])C(=O)OC</smiles>

Colourless oil

Molecular weight:

Yield:

${ }^{1} \mathrm{H}-\mathrm{NMR}\left(250 \mathrm{MHz}, \mathrm{CDCl}_{3}\right)$

${ }^{13} \mathrm{C}-\mathrm{NMR}\left(63 \mathrm{MHz}, \mathrm{CDCl}_{3}\right)$ : $\mathrm{MS}$ :

IR (neat):

$\mathrm{R}_{\mathrm{f}}$ :

Elemental analysis (\%):
$247.25\left(\mathrm{C}_{10} \mathrm{H}_{17} \mathrm{NO}_{6}\right)$

$53 \%(150 \mathrm{mg})$

$\delta=1.26(3 \mathrm{H}, \mathrm{t}, J=7.1 \mathrm{~Hz}) ; 1.56-1.80(4 \mathrm{H}, \mathrm{m}) ; 2.34(2 \mathrm{H}, \mathrm{t}$, $J=6.9 \mathrm{~Hz}) ; 3.23(1 \mathrm{H}, \mathrm{m}) ; 3.76(3 \mathrm{H}, \mathrm{s}) ; 4.13(2 \mathrm{H}, \mathrm{q}$, $J=7.1 \mathrm{~Hz}) ; 4.46(1 \mathrm{H}, \mathrm{dd}, J=14.3 \mathrm{~Hz}, 4.9 \mathrm{~Hz}) ; 4.76(1 \mathrm{H}, \mathrm{dd}$, $J=14.3 \mathrm{~Hz}, 9.2 \mathrm{~Hz}$ )

$\delta=14.2,22.0,28.6,33.6,42.7,52.5,60.6,75.0,172.4,172.8$ CI: $\mathrm{m} / \mathrm{z}=249(15 \%) ; 248(100 \%) ; 214(8 \%) ; 203(6 \%)$; 202 (9\%); 201 (37\%); 169 (6 \%); 155 (8\%)

$\widetilde{v}=2958(\mathrm{~m}), 1735(\mathrm{~s}), 1558(\mathrm{~s}), 1438(\mathrm{w}), 1378(\mathrm{~m}), 1203$ (m), $1180(\mathrm{~m}), 1031(\mathrm{~m})$

0.16 (PE:EtOAc / 6:1)

Calcd.: C 48.58; H 6.93; N 5.67

Found: C 48.56; H 6.84; N 5.67 


\section{General experimental procedure for conjugate addition of dimyrtanylzinc (10) to nitroolefins:}

Trimethylsilyl chloride $(0.19 \mathrm{~mL}, 1.53 \mathrm{mmol})$ was added dropwise at $-30{ }^{\circ} \mathrm{C}$ to a solution of nitroolefin $(1.53 \mathrm{mmol})$ and copper(II) triflate $(29 \mathrm{mg}, 0.08 \mathrm{mmol}, 5 \mathrm{~mol} \%$ referring to nitroolefin) in THF $(5 \mathrm{~mL})$. The mixture was stirred for $10 \mathrm{~min}$, and a solution of dimyrtanylzinc ${ }^{1}$ 10 (ca. $3.5 \mathrm{mmol}$, ca. $1 \mathrm{M}$ solution in THF) was added dropwise. The mixture was stirred for $4 \mathrm{~h}$ at $-30{ }^{\circ} \mathrm{C}$, diluted with THF $(10 \mathrm{~mL})$ and aqueous $\mathrm{HCl}$ solution $(10 \%, 5 \mathrm{~mL})$ was added and stirring was continued for $15 \mathrm{~min}$. The mixture was poured into a mixture containing ether $(100 \mathrm{~mL})$ and saturated aqueous $\mathrm{NH}_{4} \mathrm{Cl}(50 \mathrm{~mL})$, stirred for $20 \mathrm{~min}$ and filtered over Celite ${ }^{\circledR}$. The layers were separated. The aqueous layer was washed with ether $(2 \times 30 \mathrm{~mL})$. The combined organic layers were washed with brine $(30 \mathrm{~mL})$, dried $\left(\mathrm{MgSO}_{4}\right)$ and filtered. The solvent was evaporated, and the residual oil was purified by flash chromatography to afford the product.

\section{$(1 S, 2 S, 5 S)-6,6-D i m e t h y l-2-\left(3^{\prime}\right.$-nitro-2'-phenylpropyl)bicyclo[3.1.1]heptane (12a)}

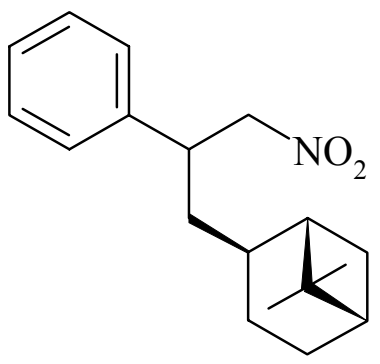

Colourless oil (mixture of diastereomers 1:1)

Molecular weight:

Yield:

${ }^{1} \mathrm{H}-\mathrm{NMR}\left(500 \mathrm{MHz}, \mathrm{CDCl}_{3}\right)$ :

${ }^{13} \mathrm{C}-\mathrm{NMR}\left(126 \mathrm{MHz}, \mathrm{CDCl}_{3}\right)$ :

MS:

IR (neat):

$\mathrm{R}_{\mathrm{f}}$ :

Elemental analysis (\%):
$287.40\left(\mathrm{C}_{18} \mathrm{H}_{25} \mathrm{NO}_{2}\right)$

$74 \%(325 \mathrm{mg})$

$\delta=0.98(6 \mathrm{H}, \mathrm{s}) ; 1.13(3 \mathrm{H}, \mathrm{s}) ; 1.20(3 \mathrm{H}, \mathrm{s}) ; 1.36(1 \mathrm{H}, \mathrm{m}) ; 1.53$

$(1 \mathrm{H}, \mathrm{m}) ; 1.64-1.98(18 \mathrm{H}, \mathrm{m}) ; 2.22(1 \mathrm{H}, \mathrm{m}) ; 2.29(1 \mathrm{H}, \mathrm{m})$;

3.47-3.57 (2H, m); 4.45-4.57 (4H, m); 7.15-7.21 (4H, m);

7.23-7.29 (2H, m); 7.30-7.36 (4H, m)

$\delta=21.3,22.8 ; 23.2,23.4 ; 26.2,26.3 ; 28.0,28.1 ; 33.3,33.6$;

$37.6,38.0 ; 38.6,38.7 ; 40.4,40.7 ; 41.2$, 41.3; 42.3, 42.4;

$44.7,47.3 ; 81.1,81.4 ; 127.5,127.7,128.9,139.2,139.7$

CI: $\mathrm{m} / \mathrm{z}=309(18 \%) ; 255(18 \%) ; 254(20 \%) ; 253(100 \%)$;

$131(10 \%) ; 110(11 \%)$

$\widetilde{v}=2910(\mathrm{~s}), 1552(\mathrm{~s}), 1495(\mathrm{w}), 1468(\mathrm{w}), 1454(\mathrm{w}), 1378$

(m), $762(\mathrm{~m}), 700(\mathrm{~m})$

Both diastereomers: 0.71 (PE:EtOAc / 10:1)

Calcd.: C 75.22; H 8.77; N 4.84

Found: C 75.32; H 9.19; N 4.84 
Ethyl 3-((1'S,2'S,5'S)-6',6'-Dimethylbicyclo[3.3.1]hept-2'-yl)-2-(nitromethyl)propanoate (12b)

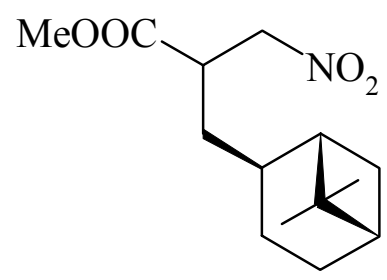

Colourless oil (mixture of diastereomers 1:1)

Molecular weight:

Yield:

${ }^{1} \mathrm{H}-\mathrm{NMR}\left(250 \mathrm{MHz}, \mathrm{CDCl}_{3}\right)$ :

${ }^{13} \mathrm{C}-\mathrm{NMR}\left(126 \mathrm{MHz}, \mathrm{CDCl}_{3}\right)$ :

MS:

$\mathrm{R}_{\mathrm{f}}$ :

IR (neat):

Elemental analysis (\%):
$269.34\left(\mathrm{C}_{14} \mathrm{H}_{23} \mathrm{NO}_{4}\right)$.

$89 \%(368 \mathrm{mg})$

$\delta=0.99(2$ signals, $6 \mathrm{H}, \mathrm{s}) ; 1.19(6 \mathrm{H}, \mathrm{s}) ; 1.39-1.50(2 \mathrm{H}, \mathrm{m})$; 1.54-1.63 (2H, m); 1.63-2.09 (16H, m); 2.30-2.41 (2H, m); 3.18-3.29 (2H, m); $3.74(3 \mathrm{H}, \mathrm{s}), 3.75(3 \mathrm{H}, \mathrm{s}) ; 4.40(1 \mathrm{H}, \mathrm{dd}$, $J=14.3 \mathrm{~Hz}, 10.4 \mathrm{~Hz}), 4.41(1 \mathrm{H}, \mathrm{dd}, J=14.3 \mathrm{~Hz}, 10.1 \mathrm{~Hz})$; $4.70(1 \mathrm{H}, \mathrm{dd}, J=14.4 \mathrm{~Hz}, 2.5 \mathrm{~Hz}),(1 \mathrm{H}, \mathrm{dd}, J=14.4 \mathrm{~Hz}$, $2.5 \mathrm{~Hz})$

$\delta=22.0,22.1 ; 23.2 ; 26.2 ; 28.0 ; 33.4,33.5 ; 36.8,37.1 ; 38.5$, $38.5 ; 38.6,38.6 ; 41.2,41.2 ; 41.4,41.5 ; 45.8,46.2 ; 52.4$, $52.5 ; 75.2,75.7 ; 173.1,173.2$

EI: $\mathrm{m} / \mathrm{z}=234(4 \%) ; 82(34 \%) ; 81(33 \%) ; 79(32 \%) ; 69$ (100\%); 67 (87\%); 55 (91\%); 41 (94\%); 18 (61\%); 15 $(37 \%)$

Both diastereomers: 0.28 (PE: $\left.\mathrm{Et}_{2} \mathrm{O} / 10: 1\right)$

$\widetilde{v}=2912$ (s, br.), 1736 (s), 1558 (s), 1439 (m), 1379 (m), $1248(\mathrm{~m}), 1203(\mathrm{~m}), 1174(\mathrm{~m})$

Calcd.: C 62.43; H 8.61; N 5.20

Found: C 62.47; H 8.70; N 5.34

\section{Experimental procedure for conjugate addition of bis[2-(ethoxycarbonyl)ethyl]zinc (11) to nitrostyrene:}

\section{Ethyl 4-Phenyl-5-nitropentanoate (13)}

$305 \mathrm{mg}$ of anhydrous zinc chloride (ca. $2.24 \mathrm{mmol}$ ) were heated to the melting point in vacuo (ca. $0.01 \mathrm{mbar})$. The residue was weighed $(285 \mathrm{mg}, 2.09 \mathrm{mmol})$, ether $(9 \mathrm{~mL})$ was added, and the mixture was sonicated until dissolution of the salt. 1-Ethoxy-1-(trimethylsilyloxy)cyclopropane ${ }^{2}$ $(0.91 \mathrm{~g}, 5.22 \mathrm{mmol})$ was added, and the mixture was stirred for $4 \mathrm{~h}$ at RT. About 6-7 $\mathrm{mL}$ of ether were removed under reduced pressure, and the residual solution was diluted with $8 \mathrm{~mL}$ of hexane. The supernatant liquid was separated, and the solvent was removed in vacuo to give bis[2-(ethoxycarbonyl)ethyl]zinc 11 as an oil. Ether $(3 \mathrm{~mL})$ was added, and the mixture was added dropwise at $-40{ }^{\circ} \mathrm{C}$ to a solution of nitrostyrene (155 mg, $\left.1.04 \mathrm{mmol}\right)$ and copper(II) triflate ( $8 \mathrm{mg}, 0.02 \mathrm{mmol}, 2 \mathrm{~mol} \%$ referring to nitroolefin) in ether $(5 \mathrm{~mL})$. The reaction mixture was 
allowed to warm up to $0{ }^{\circ} \mathrm{C}$ overnight. It was diluted with ether $(20 \mathrm{~mL})$ and quenched by addition of a saturated aqueous $\mathrm{NH}_{4} \mathrm{Cl}$ solution $(20 \mathrm{~mL})$. The aqueous layer was extracted with ether $(3 \times 30 \mathrm{~mL})$. The combined organic layers were washed with brine $(20 \mathrm{~mL})$, dried $\left(\mathrm{MgSO}_{4}\right)$, and filtered. The solvent was evaporated, and the residual oil was purified by flash chromatography (pure PE) to afford the product.<smiles>CCOC(=O)CCC(C[N+](=O)[O-])c1ccccc1</smiles>

Colourless oil

Molecular weight:

$251.28\left(\mathrm{C}_{13} \mathrm{H}_{17} \mathrm{NO}_{4}\right)$

Yield: $65 \%(170 \mathrm{mg})$

${ }^{1} \mathrm{H}-\mathrm{NMR}\left(250 \mathrm{MHz}, \mathrm{CDCl}_{3}\right): \quad \delta=1.21(3 \mathrm{H}, \mathrm{t}, J=7.2 \mathrm{~Hz}) ; 1.88-2.24(4 \mathrm{H}, \mathrm{m}) ; 3.49(1 \mathrm{H}, \mathrm{m})$; $4.08(2 \mathrm{H}, \mathrm{q}, J=7.2 \mathrm{~Hz}) ; 4.51-4.64(2 \mathrm{H}, \mathrm{m}) ; 7.12-7.43(5 \mathrm{H}$, $\mathrm{m})$

${ }^{13} \mathrm{C}-\mathrm{NMR}\left(63 \mathrm{MHz}, \mathrm{CDCl}_{3}\right): \quad \delta=14.2,28.1,31.7,43.7,60.6,80.6,127.6,128.0,129.1$, $138.3,172.5$

MS:

CI: $\mathrm{m} / \mathrm{z}=252(10 \%) ; 219(14 \%) ; 218(100 \%) ; 172(11 \%)$

IR (neat): $\widetilde{v}=2981(\mathrm{w}), 1731(\mathrm{~s}), 1552(\mathrm{~s}), 1454(\mathrm{~m}), 1378(\mathrm{~m}), 1182$ (m), $1159(\mathrm{~m}), 1037(\mathrm{~m}), 765(\mathrm{w}), 701(\mathrm{~m})$

Rf: 0.35 (PE:EtOAc / 7:1)

Elemental analysis (\%): Calcd.: C 62.14; H 6.82; N 5.57

Found: C 62.19; H 6.89; N 5.60

(1) For synthesis of dimyrtanylzinc, see: (a) Langer, F.; Devasagayaraj, A.; Chavant, P.-Y.; Knochel, P. Synlett 1994, 410. (b) The Practical Approach in Chemistry Series, Knochel, P.; Jones, P.; Langer, F.; Organozinc reagents : a practical approach; Knochel, P.; Jones, P.; Eds.; Oxford: University Press; 1999, P. 167-168.

(2) For synthesis of 1-ethoxy-(1-trimethylsilyloxy)cyclopropane, see: Rühlmann, K.; Synthesis 1971, 5, $236-253$. 